\title{
Collision-Induced Broadband Optical Nonreciprocity
}

\author{
Chao Liang, ${ }^{1}$ Bei Liu, ${ }^{1}$ An-Ning Xu, ${ }^{1}$ Xin Wen, ${ }^{1}$ Cuicui Lu $\odot,{ }^{2,3}$ Keyu Xia $\odot,{ }^{4}$ Meng Khoon Tey, ${ }^{1,5}$ \\ Yong-Chun Liu $\odot,{ }^{1,5, *}$ and $\mathrm{Li} \mathrm{You}^{1,5}$ \\ ${ }^{1}$ State Key Laboratory of Low Dimensional Quantum Physics, Department of Physics, Tsinghua University, Beijing 100084, China \\ ${ }^{2}$ Key Laboratory of Advanced Optoelectronic Quantum Architecture and Measurements of Ministry of Education, \\ Beijing Key Laboratory of Nanophotonics and Ultrafine Optoelectronic Systems, School of Physics, \\ Beijing Institute of Technology, Beijing 100081, China \\ ${ }^{3}$ Collaborative Innovation Center of Light Manipulations and Applications, Shandong Normal University, Jinan 250358, China \\ ${ }^{4}$ College of Engineering and Applied Sciences, Nanjing University, Nanjing 210093, China \\ ${ }^{5}$ Frontier Science Center for Quantum Information, Beijing 100084, China
}

(Received 30 April 2020; revised 28 July 2020; accepted 17 August 2020; published 14 September 2020)

\begin{abstract}
Optical nonreciprocity is an essential property for a wide range of applications, such as building nonreciprocal optical devices that include isolators and circulators. The realization of optical nonreciprocity relies on breaking the symmetry associated with Lorentz reciprocity, which typically requires stringent conditions such as introducing a strong magnetic field or a high-finesse cavity with nonreciprocal coupling geometry. Here we discover that the collision effect of thermal atoms, which is undesirable for most studies, can induce broadband optical nonreciprocity. By exploiting the thermal atomic collision, we experimentally observe magnet-free and cavity-free optical nonreciprocity, which possesses a high isolation ratio, ultrabroad bandwidth, and low insertion loss simultaneously. The maximum isolation ratio is close to $40 \mathrm{~dB}$, while the insertion loss is less than $1 \mathrm{~dB}$. The bandwidth for an isolation ratio exceeding $20 \mathrm{~dB}$ is over $1.2 \mathrm{GHz}$, which is 2 orders of magnitude broader than typical resonance-enhanced optical isolators. Our work paves the way for the realization of high-performance optical nonreciprocal devices and provides opportunities for applications in integrated optics and quantum networks.
\end{abstract}

DOI: 10.1103/PhysRevLett.125.123901

Optical nonreciprocity, arising from breaking the Lorentz reciprocity theorem, has been a fundamental topic in optics and electromagnetics for centuries [1]. It plays an important role in optical devices such as isolators and circulators, which are indispensable for applications ranging from optical signal processing [2,3] to quantum networks [4,5]. The traditional method of realizing optical nonreciprocity is based on the magneto-optical Faraday effect [6-8], but such devices are not convenient for integration due to problems such as crosstalk caused by the magnetic field and lattice mismatches between magneto-optic materials and silicon [9]. Therefore, in recent years magnet-free nonreciprocity has attracted growing interest and led to many studies. These include examinations of it in relation to nonlinear effects $[10,11]$, the dynamic modulation of permittivity [12-14], optomechanical interactions [15-19], interfering parametric processes [20,21], chiral photonic crystal waveguides [22], PT symmetry enhanced nonlinearity [23,24], synthetic

Published by the American Physical Society under the terms of the Creative Commons Attribution 4.0 International license. Further distribution of this work must maintain attribution to the author(s) and the published article's title, journal citation, and DOI. magnetism [25-29], cold atomic Bragg lattices [30-33], hot atoms [34-36], and spinning cavities [37,38].

Despite the rapid progress in this area, it remains a major challenge to realize optical nonreciprocity with a high isolation ratio, broad bandwidth, and low insertion loss simultaneously. In previous studies, many approaches make use of optical cavities [39-47], which can realize a high isolation ratio due to the cavity-enhanced interaction, but the bandwidth is inherently limited by the cavity linewidth, typically less than $10 \mathrm{MHz}$. Other schemes achieve a broad isolation bandwidth, but the isolation ratios are relatively low due to relatively weak interactions $[48,49]$.

Here we experimentally realize high-performance magnet-free and cavity-free optical nonreciprocity, which simultaneously possesses an ultrahigh isolation ratio and ultrabroad bandwidth, as well as low insertion loss. By taking advantage of the random motion and collision of thermal atoms, we realize direction-dependent population trapping of the atoms, leading to the nonreciprocal behavior for the probe laser passing through the atoms. By combining multiple resonance enhancements, the isolation ratio reaches as high as $40 \mathrm{~dB}$, the bandwidth for isolation ratios exceeding $20 \mathrm{~dB}$ is over $1.2 \mathrm{GHz}$, and the insertion loss is less than $1 \mathrm{~dB}$. These results represent a bandwidth that is over 2 orders of magnitude broader than typical 


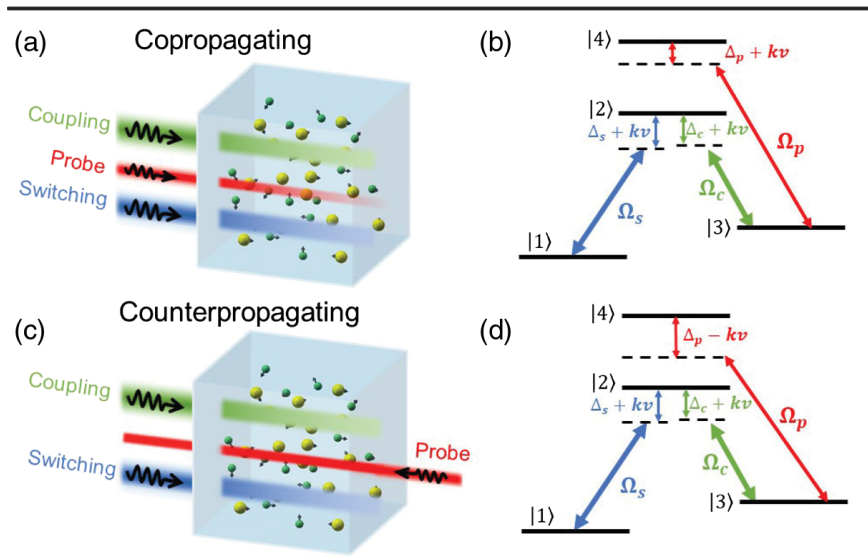

FIG. 1. Schematic diagram of the experiments [(a) and (c)] and the corresponding atoms' energy level diagrams [(b) and (d)]. In (a) and (c), the cube represents the atomic vapor cell, and the rubidium (buffer gas) atoms are illustrated by yellow (green) dots in random motion along the direction of attached arrows. The switching laser (blue) and coupling laser (green) propagate in the same direction, while the probe laser (red) can be either copropagating (a) or counterpropagating (c). In the experiments, the three laser beams are spatially overlapped in the cell.

resonance-enhanced optical isolators. In addition, the dynamic range of isolated light power spans more than 1 order of magnitude. Thus, this Letter opens up realistic opportunities for practical applications in optical signal processing.

The schematic diagram for our experimental setup is shown in Figs. 1(a) and 1(c). A cubic glass cell (side length $1 \mathrm{~cm})$ is filled with gaseous rubidium-87 $\left({ }^{87} \mathrm{Rb}\right)$ atoms and buffer gas (helium-4 and nitrogen). The pressure of the buffer gas is 30 Torr, and the temperature of the cell is stabilized at $75^{\circ} \mathrm{C}$ unless otherwise specified. Three laser beams are used: switching and coupling lasers for controlling the atoms and a probe laser for examining optical nonreciprocity. The propagation directions of the switching and coupling lasers are the same, while the prorogation direction of the probe laser can be the same as (copropagating case) or opposite to (counterpropagating case) the switch and coupling lasers. The polarization of the probe laser is perpendicular to the polarization of the switching and coupling lasers so that the transmitted signal of the probe laser can be separately measured.

The energy level diagrams of the atoms are shown in Figs. 1(b) and 1(d) for the copropagating and counterpropagating cases, respectively. The switching, coupling, and probe lasers drive the transitions $\left|5^{2} S_{1 / 2}, F=1\right\rangle \Leftrightarrow$ $\left|5^{2} P_{1 / 2}, F=1\right\rangle(|1\rangle \Leftrightarrow|2\rangle), \quad\left|5^{2} S_{1 / 2}, F=2\right\rangle \Leftrightarrow\left|5^{2} P_{1 / 2}, F=1\right\rangle$ $(|3\rangle \Leftrightarrow|2\rangle)$, and $\left|5^{2} S_{1 / 2}, F=2\right\rangle \Leftrightarrow\left|5^{2} P_{1 / 2}, F=2\right\rangle(|3\rangle \Leftrightarrow|4\rangle)$, respectively. Their Rabi frequencies and wave numbers are correspondingly denoted by $\left(\Omega_{s}, \Omega_{c}, \Omega_{p}\right)$ and $\left(k_{s}, k_{c}, k_{p}\right)$. The detunings of the three lasers from their respectively coupled transitions are denoted by $\left(\Delta_{s}, \Delta_{c}, \Delta_{p}\right)$. For atoms moving with a velocity $v$, the detunings change into
$\left(\Delta_{s}+k_{s} v, \Delta_{c}+k_{c} v, \Delta_{p}+k_{p} v\right)$ for the copropagating case and $\left(\Delta_{s}+k_{s} v, \Delta_{c}+k_{c} v, \Delta_{p}-k_{p} v\right)$ for the counterpropagating case due to Doppler shift. In the rotating reference frame and undepleted control field approximation, the Hamiltonian for this group of atoms reads $H_{\mathrm{co} / \mathrm{cou}}(v)=-\left(\Delta_{s}+k_{s} v\right) \sigma_{11}-\left(\Delta_{c}+k_{c} v\right) \sigma_{33}+\left(\Delta_{p}-\right.$ $\left.\Delta_{c} \pm k_{p} v-k_{c} v\right) \sigma_{44}+\frac{1}{2}\left(\Omega_{s} \sigma_{21}+\Omega_{c} \sigma_{23}+\Omega_{p} \sigma_{43}+\right.$ H.c. $)$, where $\sigma_{m n}=|m\rangle\langle n|(n, m=1,2,3,4)$ are the atomic transition operators. Here the difference between $H_{\text {co }}$ and $H_{\text {cou }}$ for copropagating and counterpropagating is the term $\pm k_{p} v \sigma_{44}$, with + for the former and - for the latter. Since the frequency differences among the three lasers are quite small compared to their absolute frequencies, $k_{s}, k_{c}$, and $k_{p}$ are essentially equal and can be uniformly denoted by $k$. By solving the master equation $\dot{\rho}=-i\left[H_{\mathrm{co} / \mathrm{cou}}, \rho\right]+$ $\mathcal{L}[\rho]$ with $\mathcal{L}[\rho]$ being the Lindblad operator describing the decay and dephasing of the atoms, we can calculate the stationary density matrix elements and obtain the transmission for the probe laser [50]. To understand the emergence of nonreciprocity, we focus on the atomic velocity distribution in level $|3\rangle$

$\tilde{\rho}_{33}^{(\mathrm{co} / \mathrm{cou})}(v)=f(v) \rho_{33}\left(\Delta_{s}+k v, \Delta_{c}+k v, \Delta_{p} \pm k v\right)$,

where $f(v)=\exp \left(-v^{2} / u^{2}\right) /(\sqrt{\pi} u)$ is the Maxwellian velocity distribution, $u=\sqrt{2 k_{B} T / m}$ is the most probable speed of atoms, $k_{B}$ is the Boltzmann constant, $T$ is the temperature of the atomic gas, and $m$ is the mass of the atom. In the absence of the switching and coupling lasers, the system is reciprocal because the atomic velocity distributions are symmetric for forward and backward directions. The presence of switching and coupling lasers breaks such a symmetry. Then, $\tilde{\rho}_{33}^{\text {co }}(v)$ and $\tilde{\rho}_{33}^{\text {cou }}(v)$ become quite different, leading to optical nonreciprocity for the probe laser (see the Supplemental Material [50] for more details).

The experimental results for the typical transmission spectra of the probe laser are plotted in Fig. 2(a). It shows that the probe field exhibits a high transmittance in the counterpropagating case ( $T_{\text {cou }}$, red dots), while it is strongly absorbed in the copropagating case ( $T_{\mathrm{co}}$, blue dots). These experimental results are in good agreement with the theoretical curves plotted in Fig. 2(b) (details in the Supplemental Material [50]). To quantitatively describe the optical nonreciprocity, we introduce the isolation ratio $10 \log _{10}\left(T_{\text {cou }} / T_{\text {co }}\right)$ and contrast ratio $\left(T_{\text {cou }}-T_{\text {co }}\right) /\left(T_{\text {cou }}+T_{\text {co }}\right)$. As shown in Fig. 2(c), the maximum isolation ratio reaches nearly $40 \mathrm{~dB}$ (green dots), corresponding to a contrast ratio of 0.9999 (pink dots).

As revealed in Fig. 2, the isolation works in a remarkably broad frequency range. For convenience, we define the $20 \mathrm{~dB}$ isolation bandwidth, which corresponds to the frequency range where the isolation ratio is larger than $20 \mathrm{~dB}$ (with a contrast ratio larger than 0.99). Our typical 

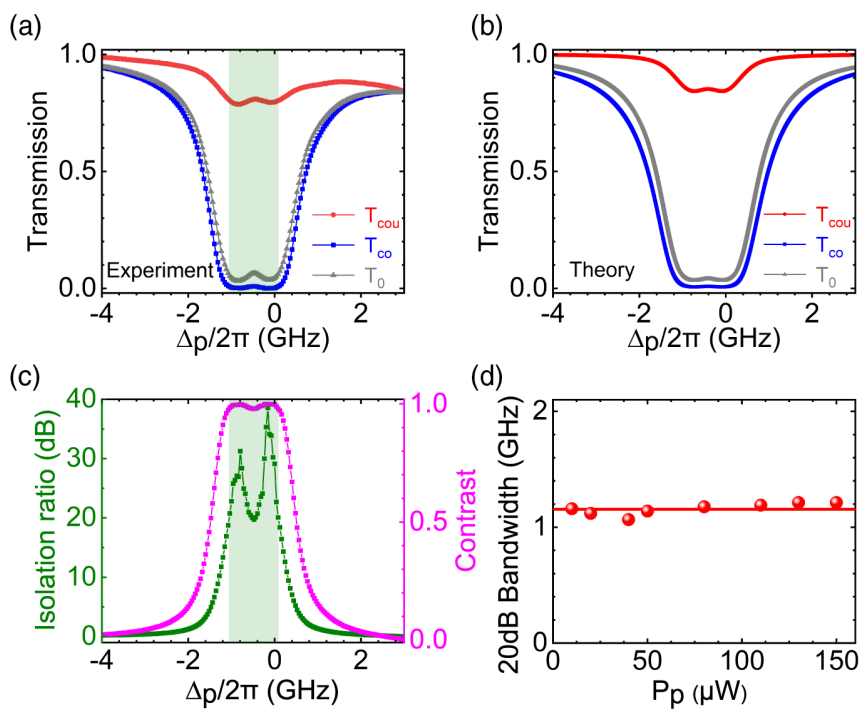

(d)

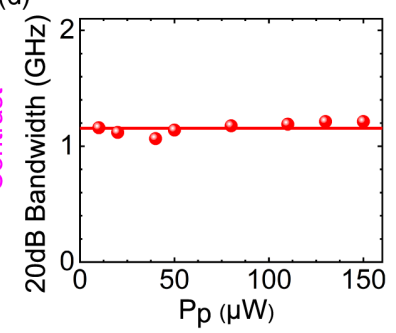

FIG. 2. (a) Experimental results for the probe laser transmission spectra in counterpropagating cases ( $T_{\text {cou }}$, red dots), copropagating ( $T_{\text {co }}$, blue dots) cases, and without the switching and coupling lasers ( $T_{0}$, gray dots). (b) Theoretical results corresponding to (a). (c) Isolation ratio (green dots, left vertical axis) and transmission contrast ratio (pink dots, right vertical axis) versus detuning of the probe field. (d) The bandwidth for isolation ratios greater than $20 \mathrm{~dB}$ [denoted by shaded regions in (a) and (c)] versus the probe laser power $P_{p}$. In (a)-(c), the switching laser power is $P_{s}=2.2 \mathrm{~mW}$, the coupling laser power is $P_{c}=1.5 \mathrm{~mW}$, and the detunings are 0 . In (d), the switching and coupling laser powers are optimized according to different probe laser powers.

experimental results show that this bandwidth exceeds 1.2 GHz [shaded regions in Figs. 2(a) and 2(c)], which is more than 200 times broader than the atomic natural linewidth. This ultrabroadband nonreciprocity originates from two aspects: atomic collision and multiple resonance enhancements. First, unlike typical experiments where atomic collisions often bring in detrimental effects, here we fill the vapor cell with buffer gas to enhance the collision between the rubidium atoms and the buffer gas atoms. This collision destroys the atomic coherence, which makes the atoms relax more rapidly. When the probe laser interacts with the atoms, the absorption linewidth becomes broader. This also leads to the broadening of the nonreciprocal bandwidth as long as this broadening is still comparable to the Doppler linewidth. In our experiments with 30 Torr buffer gas and a temperature of $75^{\circ} \mathrm{C}$, the Doppler broadening is about $540 \mathrm{MHz}$, while the collision broadening is around $570 \mathrm{MHz}$ [51]. In this case, the bandwidth can be strongly broadened while the high isolation ratio is preserved. Further increasing the buffer gas density and the temperature leads to an even broader isolation bandwidth (see Supplemental Material [50] for more results). Second, we have made use of multiple resonance enhancements to further increase the isolation bandwidth. The energy levels $|2\rangle$ and $|4\rangle$ correspond to the hyperfine splitting energy levels $\left|5^{2} P_{1 / 2}, F=1\right\rangle$ and $\left|5^{2} P_{1 / 2}, F=2\right\rangle$ of ${ }^{87} \mathrm{Rb}$ atoms, which are separated by $814 \mathrm{MHz}$. Due to the collision induced broadening, the nonreciprocal bandwidth becomes comparable to this energy level separation. Therefore, the nonreciprocal windows for these two energy levels overlap and merge, leading to the formation of a broadened nonreciprocal window. As shown in Figs. 2(a)-(c), the separation between the two dips (peaks) is about $0.8 \mathrm{GHz}$, which exactly matches the hyperfine splitting above, showing that the two dips (peaks) correspond to two resonance transitions, $|3\rangle \Leftrightarrow|4\rangle$ and $|3\rangle \Leftrightarrow|2\rangle$.

Moreover, as shown in Fig. 2(a), the typical transmittance observed within the $20 \mathrm{~dB}$ isolation bandwidth exceeds 0.8 , corresponding to an insertion loss less than $1 \mathrm{~dB}$ [50]. These properties of a high isolation ratio, ultrabroad bandwidth, and low insertion loss can also persist for a significant range of probe laser power-from 10 to $150 \mu \mathrm{W}$-as plotted in Fig. 2(d) (see Supplemental Material [50] for more results). Note that our scheme is void of the dynamic reciprocity problem $[10,52]$ because the nonreciprocity realized here originates from the atomic motion, which breaks the time reversal symmetry, instead of directly from the nonlinearity of the atoms. This feature has great potential for practical applications.

In Fig. 3, we display the measured probe laser transmission spectra for copropagating and counterpropagating settings at different control laser powers. As we fix the switching laser power at $P_{s}=2.2 \mathrm{~mW}$ and increase the coupling laser power $P_{c}$, the copropagating transmission keeps near 0 and changes only slightly [Fig. 3(a)], while the counterpropagating transmission increases strongly from 0 to 0.8 [Fig. 3(b)]. This finding reveals that the coupling laser has little effect on the transmission of the probe laser for the copropagating case but strongly influences the counterpropagating case. The increase of the counterpropagating transmission originates from the fact that a stronger coupling laser drives more atoms from state $|3\rangle$ to state $|1\rangle$ and thus the absorption for the probe laser becomes weaker. On the other hand, as we fix the coupling laser power at $P_{c}=2.4 \mathrm{~mW}$ and increase the switching laser power $P_{s}$, the copropagating transmission decreases strongly from approximately 0.8 to 0 [Fig. 3(c)], while the counterpropagating transmission keeps near 0.8 and changes only slightly [Fig. 3(d)]. This finding shows that the switching laser has a strong (small) effect on the copropagating (counterpropagating) case, which is just opposite to the coupling laser's effect. The reason is that the switching laser drives atoms from state $|1\rangle$ to state $|3\rangle$, which plays an opposite role compared with the coupling laser. Note that the control laser power required to obtain high-performance nonreciprocity is less than $3 \mathrm{~mW}$, which is practical for applications. Meanwhile, the nonreciprocity does not strictly rely on the detuning of the switching and coupling lasers, which can tolerate errors in the $\mathrm{GHz}$ range 

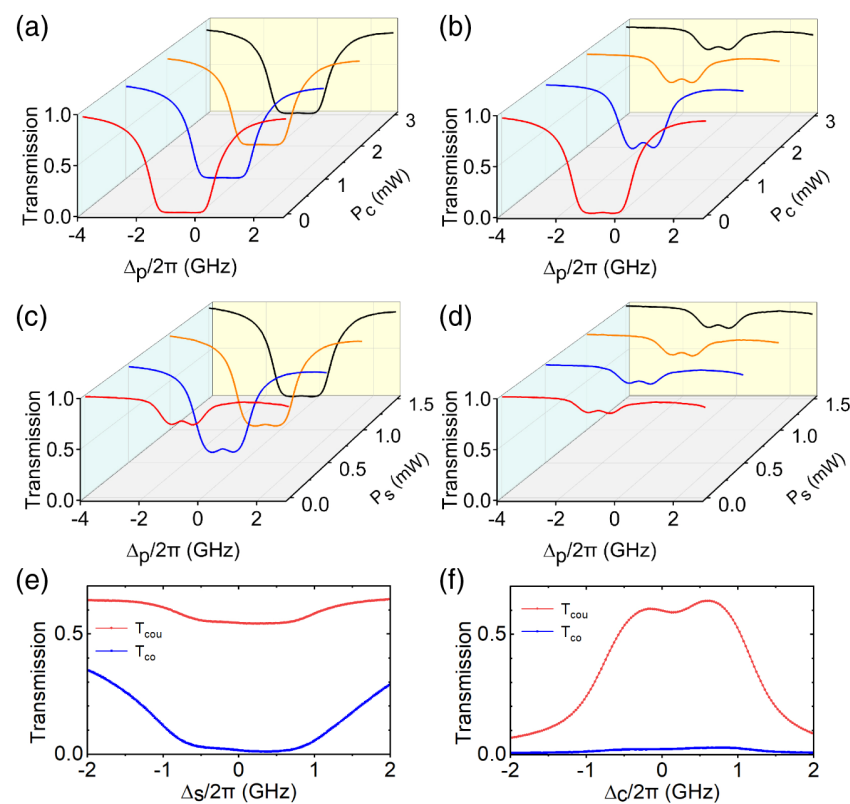

FIG. 3. (a),(b) Transmission spectra of the probe laser for different coupling laser power $P_{c}$ from 0 to $3 \mathrm{~mW}$ in the copropagating (a) and counterpropagating (b) cases. The switching laser power is fixed at $P_{s}=2.2 \mathrm{~mW}$. (c),(d) Transmission spectra of the probe laser for different switching laser power $P_{s}$ from 0 to $1.5 \mathrm{~mW}$ in the copropagating (c) and counterpropagating (d) cases. The coupling laser power is fixed at $P_{c}=2.4 \mathrm{~mW}$. (e),(f) Typical transmission of the probe laser versus the switching laser detuning $\Delta_{s}$ (e) and the control laser detuning $\Delta_{c}$ (f) in the copropagating (blue dots) and counterpropagating (red dots) cases.

[Figs. 3(e) and 3(f)], revealing that our scheme is robust to system parameter changes.

Furthermore, our system is also capable of realizing bidirectional nonreciprocity. As shown in Figs. 4(a) and 4(b), with other experimental conditions fixed, as we sweep the probe laser detuning from -5 to $10 \mathrm{GHz}$, two nonreciprocal windows appear. In addition to the first nonreciprocal window near $\Delta_{p}=0$, which has been discussed previously, a second nonreciprocal window appears near $\Delta_{p} / 2 \pi=6.8 \mathrm{GHz}$. The response of the copropagating and counterpropagating probe fields for the second nonreciprocal window becomes opposite to that of the first nonreciprocal window, i.e., the copropagating probe laser can pass through while the counterpropagating probe laser is absorbed. This bidirectional nonreciprocity originates from the different coupling routes for the probe laser with different laser frequencies. As sketched in Figs. 4(c) and $4(\mathrm{~d})$, the first nonreciprocity window is related to the transition $|3\rangle \Leftrightarrow|2\rangle$ and $|3\rangle \Leftrightarrow|4\rangle$, while the second nonreciprocity window is associated with the transition $|1\rangle \Leftrightarrow$ $|2\rangle$ and $|1\rangle \Leftrightarrow|4\rangle$. The hyperfine splitting of the two energy levels, $\left|5^{2} S_{1 / 2}, F=1\right\rangle(|1\rangle)$ and $\left|5^{2} P_{1 / 2}, F=2\right\rangle(|3\rangle)$ of ${ }^{87} \mathrm{Rb}$ atoms, is $6.8 \mathrm{GHz}$, which exactly matches the distance of the two nonreciprocity windows. Since the roles of
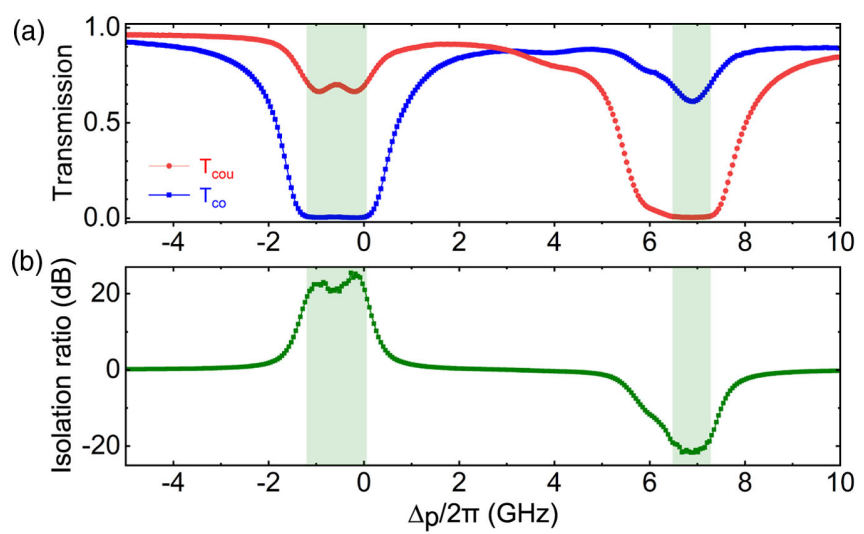

(c)

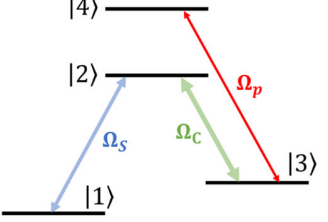

(d)

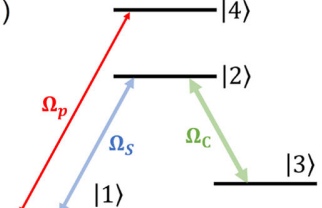

FIG. 4. (a) Transmission spectra of probe laser for the copropagating (blue dotes) and counterpropagating (red dots) cases with the probe laser detuning sweeping from -5 to $10 \mathrm{GHz}$. (b) The corresponding isolation ratio of (a). The shaded regions denote the two nonreciprocal windows with isolation ratios greater than $20 \mathrm{~dB}$. The switching laser power is $P_{s}=2.2 \mathrm{~mW}$, the coupling laser power is $P_{c}=2 \mathrm{~mW}$, and the detunings are 0. (c),(d) Energy level diagrams for the first (c) and second (d) nonreciprocal windows.

switching and coupling lasers just reverse for these two cases, it is clear that the two nonreciprocity windows just exhibit opposite properties. As shown in Fig. 4(b), we can realize high-performance nonreciprocity for both windows, with the isolation ratio greater than $20 \mathrm{~dB}$, and the isolation bandwidth exceeding $1 \mathrm{GHz}$.

It is worth noting that our approach is essentially different from the typical nonreciprocity electromagnetically induced transparency (EIT) effect [35,36], The EIT effect takes advantage of the coherence of atoms, but our mechanism makes use of strong atomic collisions, which strongly destroy the coherence. The typical nonreciprocal EIT bandwidth is on the order of $10 \mathrm{MHz}$, which is limited by the intrinsic atomic resonance linewidth, while in our experiments a nonreciprocal bandwidth of over $1.2 \mathrm{GHz}$ has been observed. Due to the distinct mechanism, the transmission line shapes in our scheme are also different from the typical EIT line shape. In addition, bidirectional nonreciprocity can be realized in our experiments by simply tuning the probe laser frequency, which is not realizable in the EIT scheme.

In summary, we have experimentally demonstrated simultaneously high isolation ratio, ultrabroad bandwidth, and low insertion loss for optical nonreciprocity in a thermal atomic vapor cell, with an isolation ratio close to $40 \mathrm{~dB}$, a $20 \mathrm{~dB}$ isolation bandwidth exceeding $1.2 \mathrm{GHz}$, and an insertion loss less than $1 \mathrm{~dB}$. Remarkably, the 
operating bandwidth represents an improvement of over 2 orders of magnitude compared to the typical resonanceenhanced optical isolators. This result originates mainly from the atomic collision effect, which is usually regarded as an undesirable process for most experiments. Aided by multiple resonance enhancements, the bandwidth is further broadened, while the high isolation ratio is still maintained. Furthermore, our scheme is promising for miniaturization and integration using atomic cladding waveguides on a chip [53-58] or hollow-core photonic crystal fibers [59,60]. Therefore, our study paves the way for realizing highperformance nonreciprocal devices with potential applications in integrated optics and quantum networks, and it may also inspire the exploration of the potential influence of the anomalous Doppler effect $[61,62]$ on the nonreciprocal phenomenon.

This work is supported by the Key-Area Research and Development Program of Guangdong Province (Grant No. 2019B030330001), the National Natural Science Foundation of China (NSFC) (Grants No. 91736106, No. 11674390, No. 91836302, No. 91850117, No. 11874212, No. 11890704, No. 11654003, and No. 11654001), and the National Key R\&D Program of China (Grants No. 2018YFA0306504, No. 2019YFA0308704, and No. 2017YFA0303703), and Beijing Institute of Technology Research Fund Program for Young Scholars.

C. L. and B. L. contributed equally to this work.

${ }^{*}$ Corresponding author. ycliu@tsinghua.edu.cn

[1] H. A. Lorentz, Amsterdammer Akademie der Wetenschappen 4, 176 (1896), https://www.lorentz.leidenuniv.nl/ILpublications/sources/Lorentz_KNAW_1895.pdf.

[2] D. Jalas, A. Petrov, M. Eich, W. Freude, S. Fan, Z. Yu, R. Baets, M. Popović, A. Melloni, J. D. Joannopoulos, M. Vanwolleghm, C. R. Doerr, and H. Renner, Nat. Photonics 7, 579 (2013).

[3] C. Caloz, A. Alù, S. Tretyakov, D. Sounas, K. Achouri, and Z. L. Deck-Léger, Phys. Rev. Applied 10, 047001 (2018).

[4] H. Kimble, Nature (London) 453, 1023 (2008).

[5] P. Lodahl, S. Mahmoodian, S. Stobbe, P. Schneeweiss, J. Volz, A. Rauschenbeutel, H. Pichler, and P. Zoller, Nature (London) 541, 473 (2017).

[6] Z. Wang, Y. Chong, J. D. Joannopoulos, and M. Soljačić, Nature (London) 461, 772 (2009).

[7] A. B. Khanikaev, S.H. Mousavi, G. Shvets, and Y.S. Kivshar, Phys. Rev. Lett. 105, 126804 (2010).

[8] L. Bi, J. Hu, P. Jiang, D. H. Kim, G. F. Dionne, L. C. Kimerling, and C. A. Ross, Nat. Photonics 5, 758 (2011).

[9] D. Dai, J. Bauters, and J. E. Bowers, Light Sci. Appl. 1, e1 (2012).

[10] A. B. Khanikaev and A. Alù, Nat. Photonics 9, 359 (2015).
[11] D. L. Sounas, J. Soric, and A. Alù, National Electron. Rev. 1, 113 (2018).

[12] Z. Yu and S. Fan, Nat. Photonics 3, 91 (2009).

[13] N. A. Estep, D. L. Sounas, J. Soric, and A. Alù, Nat. Phys. 10, 923 (2014).

[14] M. S. Kang, A. Butsch, and P. S. J. Russell, Nat. Photonics 5, 549 (2011).

[15] M. Hafezi and P. Rabl, Opt. Express 20, 7672 (2012).

[16] F. Ruesink, M.-A. Miri, A. Alù, and E. Verhagen, Nat. Commun. 7, 13662 (2016).

[17] J. Kim, M. C. Kuzyk, K. Han, H. Wang, and G. Bahl, Nat. Phys. 11, 275 (2015).

[18] Z. Shen, Y.-L. Zhang, Y. Chen, C.-L. Zou, Y.-F. Xiao, X.-B. Zou, F.-W. Sun, G.-C. Guo, and C.-H. Dong, Nat. Photonics 10, 657 (2016).

[19] G. A. Peterson, F. Lecocq, K. Cicaka, R. W. Simmonds, J. Aumentado, and J. D. Teufel, Phys. Rev. X 7, 031001 (2017).

[20] A. Kamal, J. Clarke, and M. H. Devoret, Nat. Phys. 7, 311 (2011).

[21] A. Kamal, A. Roy, J. Clarke, and M. H. Devoret, Phys. Rev. Lett. 113, 247003 (2014).

[22] I. Söllner, S. Mahmoodian, S. L. Hansen, L. Midolo, A. Javadi, G. Kiršanské, T. Pregnolato, H. El-Ella, E. H. Lee, J. D. Song, S. Stobbe, and P. Lodahl, Nat. Nanotechnol. 10, 775 (2015).

[23] B. Peng, S. K. Özdemir, F. Lei, F. Monifi, M. Gianfreda, G. L. Long, S. Fan, F. Nori, C. M. Bender, and L. Yang, Nat. Phys. 10, 394 (2014).

[24] L. Chang, X. Jiang, S. Hua, C. Yang, J. Wen, L. Jiang, G. W. G. Li, and M. Xiao, Nat. Photonics 8, 524 (2014).

[25] K. Fang, Z. Yu, and S. Fan, Nat. Photonics 6, 782 (2012).

[26] K. Fang, J. Luo, A. Metelmann, M. H. Matheny, F. Marquardt, A. A. Clerk, and O. Painter, Nat. Phys. 13, 465 (2017).

[27] K. Fang, Z. Yu, and S. Fan, Phys. Rev. Lett. 108, 153901 (2012).

[28] L. D. Tzuang, K. Fang, P. Nussenzveig, S. Fan, and M. Lipson, Nat. Photonics 8, 701 (2014).

[29] L. Yuan, S. Xu, and S. Fan, Opt. Lett. 40, 5140 (2015).

[30] S. A. R. Horsley, J.-H. Wu, M. Artoni, and G. C. LaRocca, Phys. Rev. Lett. 110, 223602 (2013).

[31] N. Bender, S. Factor, J. D. Bodyfelt, H. Ramezani, D. N. Christodoulides, F. M. Ellis, and T. Kottos, Phys. Rev. Lett. 110, 234101 (2013).

[32] H. Ramezani, P. K. Jha, Y. Wang, and X. Zhang, Phys. Rev. Lett. 120, 043901 (2018).

[33] D.-W. Wang, H.-T. Zhou, M.-J. Guo, J.-X. Zhang, J. Evers, and S.-Y. Zhu, Phys. Rev. Lett. 110, 093901 (2013).

[34] K. Xia, F. Nori, and M. Xiao, Phys. Rev. Lett. 121, 203602 (2018).

[35] S. Zhang, Y. Hu, G. Lin, Y. Niu, K. Xia, J. Gong, and S. Gong, Nat. Photonics 12, 744 (2018).

[36] G. Lin, S. Zhang, Y. Hu, Y. Niu, J. Gong, and S. Gong, Phys. Rev. Lett. 123, 033902 (2019).

[37] R. Huang, A. Miranowicz, J.-Q. Liao, F. Nori, and H. Jing, Phys. Rev. Lett. 121, 153601 (2018).

[38] S. Maayani, R. Dahan, Y. Kligerman, E. Moses, A. U. Hassan, H. Jing, F. Nori, D. N. Christodoulides, and T. Carmon, Nature (London) 558, 569 (2018). 
[39] P. Yang, X. Xia, H. He, S. Li, X. Han, P. Zhang, G. Li, P. Zhang, J. Xu, Y. Yang, and T. Zhang, Phys. Rev. Lett. 123, 233604 (2019).

[40] Z. Shen, Y.-L. Zhang, Y. Chen, F.-W. Sun, X.-B. Zou, G.-C. Guo, C.-L. Zou, and C.-H. Dong, Nat. Commun. 9, 1797 (2018).

[41] F. Ruesink, J. Mathew, M. Miri, A. Alù, and E. Verhagen, Nat. Commun. 9, 1798 (2018).

[42] B. He, L. Yang, X. Jiang, and M. Xiao, Phys. Rev. Lett. 120, 203904 (2018).

[43] L. Tang, J. Tang, W. Zhang, G. Lu, H. Zhang, Y. Zhang, K. Xia, and M. Xiao, Phys. Rev. A 99, 043833 (2019).

[44] K. Xia, G. Lu, G. Lin, Y. Cheng, Y. Niu, S. Gong, and J. Twamley, Phys. Rev. A 90, 043802 (2014).

[45] C. Sayrin, C. Junge, R. Mitsch, B. Albrecht, D. O’Shea, P. Schneeweiss, J. Volz, and A. Rauschenbeutel, Phys. Rev. X 5, 041036 (2015).

[46] M. Scheucher, A. Hilico, E. Will, J. Volz, and A. Rauschenbeutel, Science 354, 1577 (2016).

[47] J. Ma, J. Wen, S. Ding, S. Li, Y. Hu, X. Jiang, L. Jiang, and M. Xiao, Laser Photonics Rev. 14, 1900278 (2020).

[48] L. Ranzani, S. Kotler, A. J. Sirois, M. P. DeFeo, M. Castellanos-Beltran, K. Cicak, L. R. Vale, and J. Aumentado, Phys. Rev. Applied 8, 054035 (2017).

[49] L. Mercier de Lepinay, E. Damskägg, C. F. OckeloenKorppi, and M. A. Sillanpää, Phys. Rev. Applied 11, 034027 (2019).

[50] See Supplemental Material at http://link.aps.org/ supplemental/10.1103/PhysRevLett.125.123901 for the theoretical calculation of nonreciprocal transmission, the detailed experimental setup, and more experiment results.

[51] C. Rice, K. Lapp, A. Rapp, W. S. Miller, and G. P. Perram, J. Quant. Spectrosc. Radiat. Transfer 224, 550 (2019).

[52] Y. Shi, Z. Yu, and S. Fan, Nat. Photonics 9, 388 (2015).

[53] J. Kitching, Appl. Phys. Rev. 5, 031302 (2018).

[54] L. Stern, B. Desiatov, N. Mazurski, and U. Levy, Nat. Commun. 8, 14461 (2017).

[55] R. Ritter, N. Gruhler, W. Pernice, H. Kübler, T. Pfau, and R. Löw, Appl. Phys. Lett. 107, 041101 (2015).

[56] R. Ritter, N. Gruhler, H. Dobbertin, H. Kübler, S. Scheel, W. Pernice, T. Pfau, and R. Löw, Phys. Rev. X 8, 021032 (2018).

[57] H. Schmidt and A. R. Hawkins, Appl. Phys. Lett. 86, 032106 (2005).

[58] W. Yang, D. Conkey, B. Wu, D. Yin, A. R. Hawkins, and H. Schmidt, Nat. Photonics 1, 331 (2007).

[59] M. R. Sprague, P.S. Michelberger, T. F. Champion, D. G. England, J. Nunn, X. M. Jin, W. S. Kolthammer, A. Abdolvand, P. S. J. Russell, and I. A. Walmsley, Nat. Photonics 8, 287 (2014).

[60] P. Londero, V. Venkataraman, A. R. Bhagwat, A. D. Slepkov, and A. L. Gaeta, Phys. Rev. Lett. 103, 043602 (2009).

[61] X. Shi, X. Lin, I. Kaminer, F. Gao, Z. Yang, J. D. Joannopoulos, M. Soljačić, and B. Zhang, Nat. Phys. 14, 1001 (2018).

[62] X. Lin and B. Zhang, Laser Photonics Rev. 13, 1900081 (2019). 